\title{
A New Unified Design Environment for Optimization of Electric Machines Based on Continuum Sensitivity and B-Spline Parametrization
}

\author{
Min-Ho Kim*, Hyang-Beom Lee*, Hyeong-Seok Kim** and Jin-Kyu Byun ${ }^{\dagger}$
}

\begin{abstract}
In this paper, a unified design environment is developed for the optimization of electric machines based on continuum sensitivity. For electromagnetic (EM) system analysis, COMSOL scripting environment is used. Optimization module is developed by MATLAB programming, which can be combined with COMSOL script commands. The modules are combined into one MATLAB project, and iteration process necessary for the optimization of EM system can be performed efficiently. During the design process, visual feedback of the current design status is given to the designer. In addition, the B-Spline parametrization of the nodal points is implemented to obtain smooth boundary of the device. The developed software is applied to the problem of finding uniform flux density distribution at the air gap of an electromagnet to verify its feasibility and effectiveness.
\end{abstract}

Keywords: B-Spline, COMSOL, Continuum sensitivity, Optimal design

\section{Introduction}

The ongoing problems of climate change due to greenhouse effects are forcing more countries to reduce carbon emissions in the immediate future. Thus, there is a growing need for a new breed of energy-efficient electric machines for electric vehicles and wind turbine generators, among others.

The optimal design method for electric machines has evolved with advances in computer technology and numerical analysis methods. Among the many design methods, those based on design sensitivity analysis (DSA) have gained popularity because of their accuracy in derivative calculation and efficiency in terms of computation time. DSA can be categorized into two groups according to how the differentiation of the objective function is performed: discrete DSA (DDSA) and continuum DSA (CDSA).

DDSA gathers sensitivity information from the system discretized by finite element method (FEM) and performs the optimal design [1]. In order to implement DDSA, the finite-element system matrix assembly part of the source code must be directly modified, which is not supported by most commercial electromagnetic (EM) software. Thus, DDSA is usually implemented by building custom EM solvers, which cannot utilize powerful graphical user interface (GUI) or fast matrix solvers provided by commercial EM software. As a result, a lot of the

$\dagger$ Corresponding Author: School of Electrical Engineering, Soongsil University, Korea. (jkbyun@ssu.ac.kr)

* School of Electrical Engineering, Soongsil University, Korea. (hyang@ssu.ac.kr)

** School of Electrical and Electronics Engineering, Chung-Ang University, Korea. (kimcaf2@cau.ac.kr)

Received: October 12, 2010; Accepted: October 27, 2010 development time is spent on the interface routines outside the actual optimization module, and it is difficult to apply DDSA to a wide range of practical problems.

On the other hand, CDSA enables the calculation of sensitivity from the electric or magnetic field solution of the separate EM analysis module [2]. Hence, commercial EM software can be readily coupled with optimization module, including sensitivity calculation module. In this paper, a unified optimal design environment based on CDSA was developed utilizing scripting COMSOL [3] with MATLAB. The optimization module was programmed by MATLAB commands, and EM analysis module was built with COMSOL script. They are combined into one MATLAB project, and the automated design process was achieved. The program provided visual feedback of the shape of the device during the design process to help the designer understand the current status. The design parameters necessary for optimization was put in the MS Excel input file and accessed by the program for user convenience. Additionally, B-Spline parametrization of the nodal points was implemented to avoid the zigzag shape of the boundary and obtain smooth device boundary for increased accuracy and manufacturing convenience. To verify the effectiveness of the developed design environment, it was applied to the shape design of the electromagnet to obtain uniform magnetic field distribution in the air gap.

\section{Continuum DSA Theory}

In this section, the sensitivity equation of CDSA is derived and reviewed to provide theoretical basis for the developed unified design environment. An EM system is 
shown in Fig. 1.

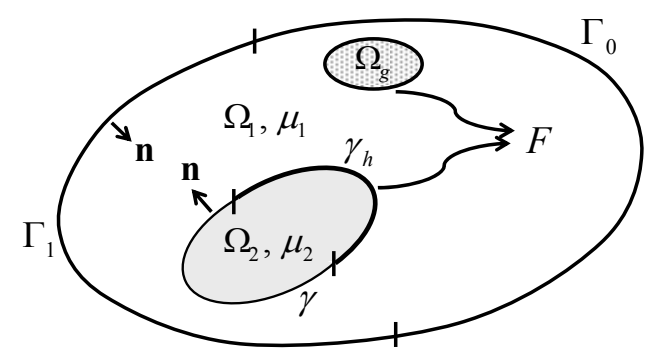

Fig. 1. Shape optimization problem in electromagnetic system

Shape optimization involves the modification of the boundary interface $\gamma$ between two different materials. Thus, the sensitivity equation with respect to the interface variation is derived. The optimization problem for electric machines can be formulated as follows:

Minimize

$$
F=\int_{\Omega} g\left(\mathbf{B}\left(\mathbf{A}_{1}\right)\right) m_{g} d \Omega+\int_{\gamma} h\left(\mathbf{B}\left(\mathbf{A}_{1}\right)\right) m_{h} d \Gamma
$$

subject to

$$
\begin{gathered}
-\nabla \times(v \mathbf{B}(\mathbf{A})-\mathbf{M})+\mathbf{J}=0 \text { in } \Omega=\Omega_{1} \cup \Omega_{2} \\
\mathbf{A}=c \quad \text { on } \Gamma_{0}, \quad \partial \mathbf{A} / \partial n=0 \quad \text { on } \Gamma_{1} \\
\mathbf{n} \cdot \mathbf{B}_{1}=\mathbf{n} \cdot \mathbf{B}_{2}, \quad \mathbf{n} \times\left(\mathbf{H}_{2}-\mathbf{H}_{1}\right)=0 \text { on } \gamma
\end{gathered}
$$

where $v$ is the magnetic reluctivity, $\mathbf{M}$ is the permanent magnetization, $\mathbf{J}$ is the current density, and the subscripts 1 and 2 denote the corresponding regions $\Omega_{1}$ and $\Omega_{2}$, respectively. The objective function $F$ is written in a general form as the sum of the area and line integrals of (1), where $g$ and $h$ are arbitrary scalar functions differentiable with respect to the magnetic flux density $\mathbf{B}(\mathbf{A})$. The characteristic functions $m_{g}$ and $m_{h}$ mark the parts of the region $\Omega$ and boundary $\gamma$ on which the objective functions are defined. The magnetic vector potential $\mathbf{A}$, which is the state variable, must satisfy the governing equation (2) and the boundary condition (3) and (4). Since the objective function $F$ is a nonlinear, implicit function of the design variable (interface variation), the augmented Lagrangian method is used to derive the sensitivity. Lagrange multiplier vector $\lambda$ is multiplied to the constraints (2)-(4) and added to $F$ in order to form the augmented version of the objective function $\bar{F}$ as follows:

$$
\begin{aligned}
\bar{F} & =\int_{\Omega 1} g\left(\mathbf{B}\left(\mathbf{A}_{1}\right)\right) m_{g} d \Omega+\int_{\gamma} h\left(\mathbf{B}\left(\mathbf{A}_{1}\right)\right) m_{h} d \Gamma \\
& -\int_{\Omega} v \mathbf{B}(\mathbf{A})^{T} \mathbf{B}(\lambda) d \Omega+\int_{\gamma}\left[\mathbf{J}^{T} \lambda+\mathbf{M}^{T} \mathbf{B}(\lambda)\right] d \Omega \\
& -\int_{\Gamma} \mathbf{H}_{t} \lambda d \Gamma
\end{aligned}
$$

where the superscript $T$ and the subscript $t$ denote the transpose and the tangential component of a vector, respectively. The integral path $\Gamma$ on the right-hand side of (5) is composed of the interface boundary $\gamma$ and the outer boundaries $\Gamma_{0}$ and $\Gamma_{1}$.

The general sensitivity equation is obtained using the variation of (5) and the concept of material derivative [2]. The final sensitivity equation is given as follows:

$$
\begin{aligned}
\dot{\bar{F}}= & \int_{\gamma}\left[\left(v_{1}-v_{2}\right) \mathbf{B}\left(\mathbf{A}_{1}\right)^{T} \mathbf{B}\left(\boldsymbol{\lambda}_{2}\right)+\boldsymbol{\lambda}_{2}\left(\mathbf{J}_{2}-\mathbf{J}_{1}\right)\right. \\
& \left.+\mathbf{B}\left(\boldsymbol{\lambda}_{2}\right)^{T} \cdot\left(\mathbf{M}_{2}-\mathbf{M}_{1}\right)-\mathbf{J}_{\mathbf{S}}{ }^{T} \mathbf{B}_{t}\left(\mathbf{A}_{1}\right) m_{h}+h H m_{h}\right] V_{n} d \Gamma
\end{aligned}
$$

where $\mathbf{J}_{s}=\nabla \times \mathbf{h}_{1}, \quad \mathbf{h}_{1}=\partial h / \partial(\mathbf{B}(\mathbf{A})), \quad \dot{\bar{F}}$ denotes the material derivate of $\bar{F}, H$ refers to the mean curvature, and $V_{n}$ is the normal component of the design velocity field $\mathbf{V}$. In (6), the Lagrange multiplier $\lambda$ is now interpreted as the adjoint variable vector, and it is defined by the state variable solution of the following adjoint system:

$$
\begin{aligned}
& -\nabla \times v \mathbf{B}(\boldsymbol{\lambda})+\left(\nabla \times \mathbf{g}_{1}\right) m_{g}=0 \text { in } \Omega \\
& \boldsymbol{\lambda}=0 \quad \text { on } \Gamma_{0}, \quad \partial \boldsymbol{\lambda} \partial n=0 \text { on } \Gamma_{1} \\
& \mathbf{n} \cdot \mathbf{B}\left(\boldsymbol{\lambda}_{1}\right)=\mathbf{n} \cdot \mathbf{B}\left(\boldsymbol{\lambda}_{2}\right), \text { and } \\
& \mathbf{n} \times\left(\mathbf{H}\left(\boldsymbol{\lambda}_{2}\right)-\mathbf{H}\left(\boldsymbol{\lambda}_{1}\right)\right)=\mathbf{J}_{s} m_{h} \text { on } \gamma
\end{aligned}
$$

where $\mathbf{g}_{1}=\partial g / \partial\left(\mathbf{B}\left(\mathbf{A}_{1}\right)\right)$ and $\mathbf{J}_{s}$ represent the adjoint sources related to the first and second integral terms of (1), respectively. Table 1 lists the adjoint sources for the magnetic system according to the different objective function types.

Table 1. Adjoint sources for the magnetic system [4]

\begin{tabular}{c|c}
\hline Type of objective function & Adjoint sources and their units \\
\hline$g=\left(B_{x i}-B_{x o}\right)^{2}$ & $\mathbf{B}_{\mathbf{r} a} \equiv \mathbf{g}_{1}=\partial g / \partial \mathbf{B}[T]$ \\
\hline$g=\left(H_{x i}-H_{x o}\right)^{2}$ & $\mathbf{M}_{a} \equiv \mathbf{g}_{1}=\partial g / \partial \mathbf{H}\left[A m^{-1}\right]$ \\
\hline
\end{tabular}

Summarizing the abovementioned steps, the state variable vector $\mathbf{A}$ and adjoint variable vector $\boldsymbol{\lambda}$ can be obtained by solving primary system (2)-(4) and adjoint system (7)-(9), respectively. $\mathbf{A}$ and $\lambda$ can then be substituted into (6) to calculate the sensitivity $\dot{\bar{F}}$.

\section{Unified Design Environment}

As shown in section 2, the calculation of sensitivity using CDSA does not require the manipulation of the system matrix equation. Thus, commercial EM software can be used to obtain the state variable solutions of the primary and adjoint systems. The calculation of the objective function and sensitivity, as well as the modification of the interface boundary, can then be taken 
care of in a compact optimization module. In this section, we use the COMSOL software as an EM analysis module and develop optimization module using MATLAB code. The two modules are then combined into one unified optimal design environment. The design variables are also parameterized using a B-Spline curve for the smoothness of the geometric contour.

\subsection{Linking COMSOL and MATLAB Using Model M-file}

Fig. 2 shows a typical modeling procedure in COMSOL where design lines and nodes are being identified. Such a modeling session can be saved as a sequence of commands [3] called a model m-file. The user can run the commands directly from the MATLAB command line or modify the model $\mathrm{m}$-file using MATLAB commands since COMSOL script is compatible with MATLAB. Thus, we can start modeling in the COMSOL user interface, then save it as a model $\mathrm{m}$-file and add the optimization module in the MATLAB environments. This process makes the modeling and analysis of the EM system very simple, enabling the designer to focus on the objective function and design variables in the optimization module. Moreover, since the adjoint system defined in (7) has the same geometry and material property as the primary system (2), we can use the same model $\mathrm{m}$-file as the primary system when solving the adjoint system. The adjoint source $\mathbf{g}_{1}=\partial g / \partial\left(\mathbf{B}\left(\mathbf{A}_{1}\right)\right)$ should be calculated in the optimization module and substituted into the model m-file using COMSOL script.

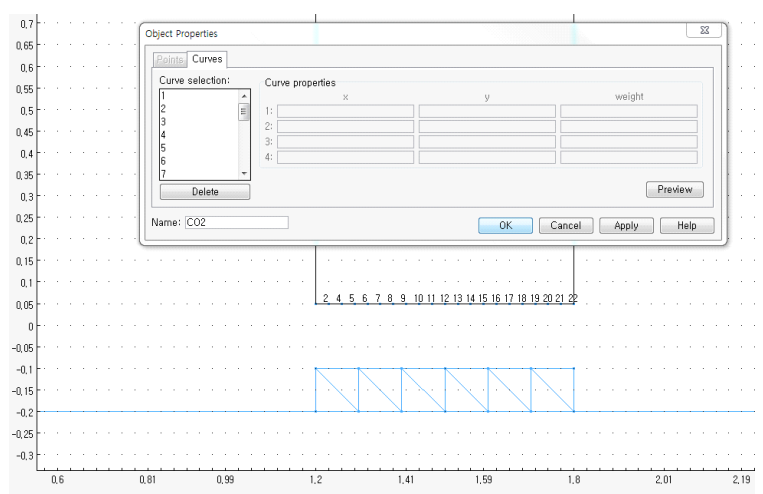

Fig. 2. Modeling procedure in COMSOL

Fig. 3 shows the flowchart of the design optimization process. The left side of the flowchart shows the analysis module utilizing COMSOL, whereas the right side represents the optimization module programmed by MATLAB codes. The data input/output between the two modules are handled by COMSOL scripting commands in the model $\mathrm{m}$-file. During the design process, the shape of the device at the present iteration is displayed using simple COMSOL script commands. They provide the designer with valuable information and insight regarding the current design status.

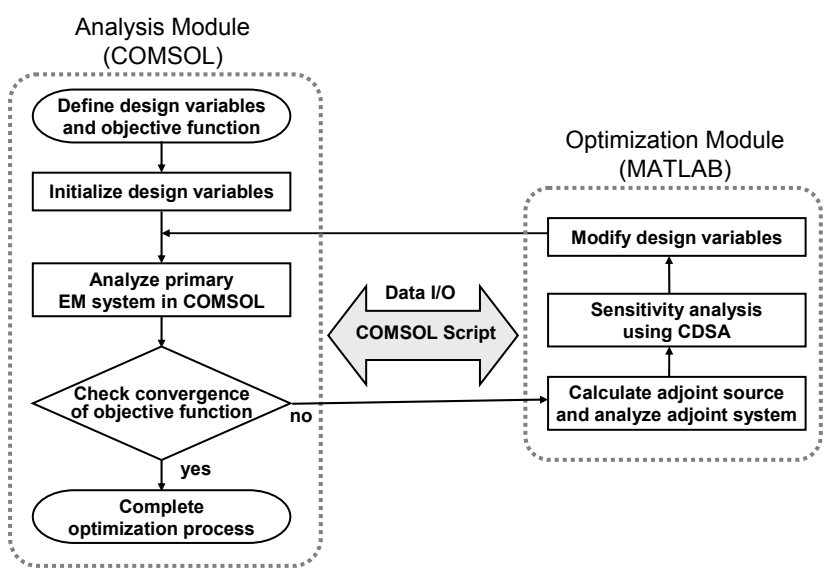

Fig. 3. Flowchart of design optimization

\subsection{B-Spline Parametrization}

In the shape design based on design sensitivity, two important points must be considered: One is the accuracy problem. If design variables have one-to-one correspondence with node points of element, the design procedure may lead to a zigzag shape, which causes serious accuracy problems near the boundary interface. The other is the manufacturing problem. If the obtained shape is too complex, it cannot be realized into a commercial product. To overcome this problem, B-Spline parameterization of node points is imposed as a design constraint.

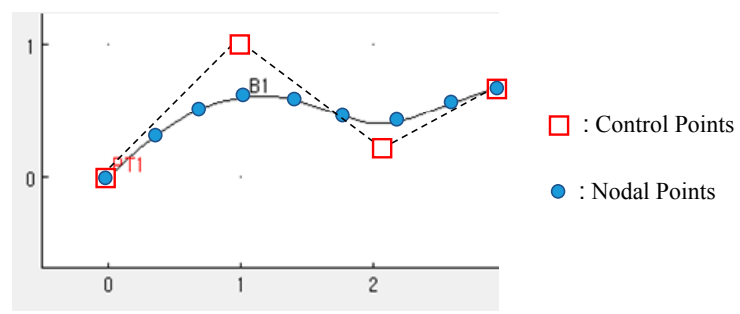

Fig. 4. Typical B-Spline curve

A typical B-Spline curve is shown in Fig. 4, where the nodal points determine the model shape to be designed. During the optimization process, only the control points are changed so that their corresponding nodal points lie on a smooth curve of B-Spline. The design variables and the control points are related by the parameterization using the B-Spline curve. This is expressed by the following:

$$
\mathbf{p}=\mathbf{N c}
$$

where $\mathbf{p}$ is a design variable vector and $\mathbf{c}$ is a control point vector. $\mathbf{N}$ is a matrix of $\mathrm{B}$-Spline parameterization and is defined as follows [5], [6]:

$$
\mathbf{N}=\left[\begin{array}{ccc}
N_{1 k}\left(t_{1}\right) & \ldots & N_{i k}\left(t_{1}\right) \\
\vdots & \ddots & \vdots \\
N_{1 k}\left(t_{j}\right) & \ldots & N_{i k}\left(t_{j}\right)
\end{array}\right]
$$


where $k$ is an order (one more than the degree), $t$ is a parameter varying typically from 0 to 1 , and $N_{i k}(t)$ is the basis function that depends on the parametric value $t$ and the order $k$ of the curve. Using (10), the sensitivity with respect to the control points can be obtained by the following:

$$
\frac{d F}{d \mathbf{c}}=\frac{d F}{d \mathbf{p}} \frac{d \mathbf{p}}{d \mathbf{c}}=\frac{d F}{d \mathbf{p}} \mathbf{N}
$$

\section{Numerical Examples}

To verify the developed unified design environment, an optimal shape design of electromagnet to obtain the uniform magnetic flux density in the objective region was tested. An MS Excel spreadsheet file was used as an input file of the design parameters for the optimization module. In order to determine the search direction and the step-size, the simple steepest descent method or the general optimizer DOT with modified feasible direction algorithm [7] can be used.

Fig. 5 shows the design variables and objective function region of the electromagnet model.

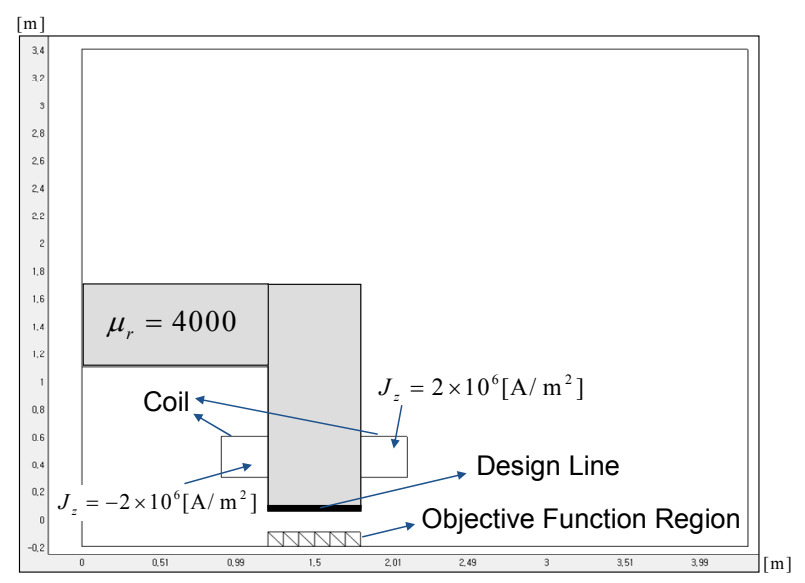

Fig. 5. Design line and objective function region of the electromagnet model

The objective function to obtain the uniform flux density distribution in the objective region is given by the following equation:

$$
F=\int_{\Omega_{g}}\left(B_{y i}-B_{y o}\right)^{2} d \Omega
$$

where $\Omega_{g}$ is the objective function region, $B_{y i}$ is the $\mathrm{y}$ component of the calculated magnetic flux density at $i$ th iteration, and $B_{y o}$ denotes the target value of $B_{y}$. In this example, $B_{y o}$ was set as -0.95 [T]. The optimization was performed in two separate cases to test the effectiveness of the B-Spline parametrization. First, 11 nodal points at the bottom side of the electromagnet were set as design variables without parametrization, as shown in Fig. 6 (case 1). Next, B-Spline parametrization was applied and 11 control points were defined at the same locations where nodal points were defined in Fig. 6 (case 2). These control points determine the B-Spline curve, and 23 nodal points were placed on that curve to achieve the smooth boundary of the magnet (the small numbers near the nodal points in Fig. 6 are the line number defined in the COMSOL).

Fig. 7 compares the final shapes of the devices of the two cases. With no parametrization (case 1), it can be observed that the magnet boundary has a zigzag shape. Whereas when B-Spline parametrization was used (case 2), the interface boundary was very smooth and practical device can be manufactured from the design. Fig. 8 shows the magnetic flux density distribution in the objective region. The final $B_{y}$ distribution was very close to the target value of $-0.95[\mathrm{~T}]$ for both cases. However, B-Spline parametrization result (case 2) shows a slightly more uniform distribution compared to case 1. Fig. 9 shows the convergence of the objective function for both cases. In the

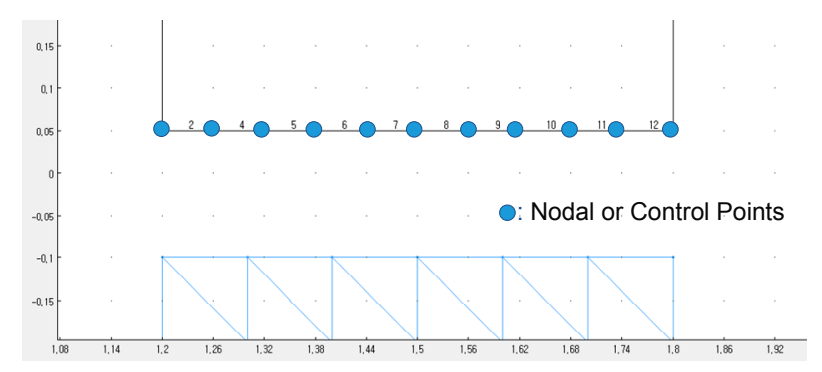

Fig. 6. Nodal or control points as design variables

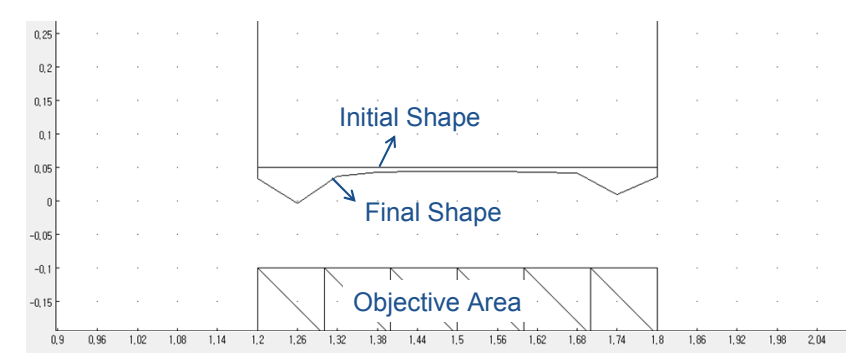

(a) No parametrization

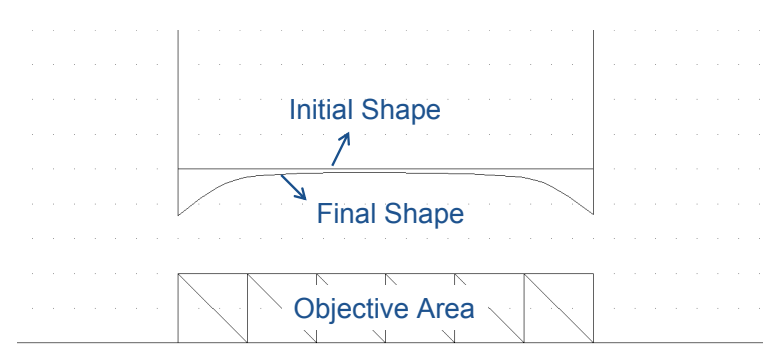

(b) B-Spline parametrization

Fig. 7. Comparison of the initial and final electromagnet shapes: 
early iterations, case 1 (nodal points) shows slightly faster convergence. However, after eight iterations, case 2 (BSpline with control points) shows faster and more stable convergence characteristics. This robustness of the BSpline parametrization was expected because it prevents the formation of the zigzag shape at the device boundary, which can lead to inaccuracy in the analysis.

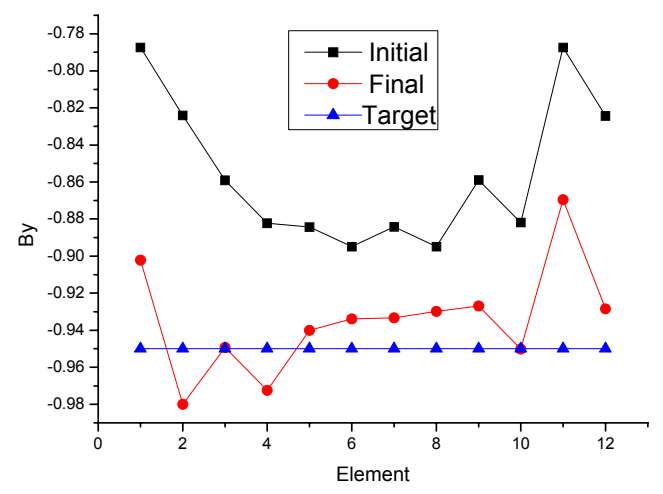

(a) No parametrization

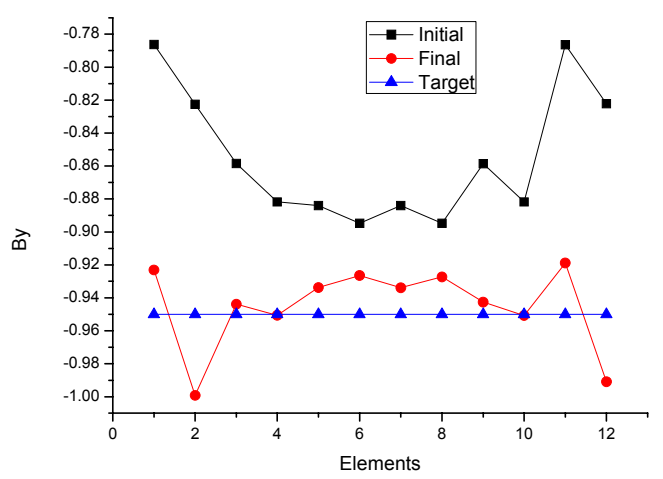

(b) B-Spline parametrization

Fig. 8. Comparison of the magnetic flux density distribution before and after optimization:

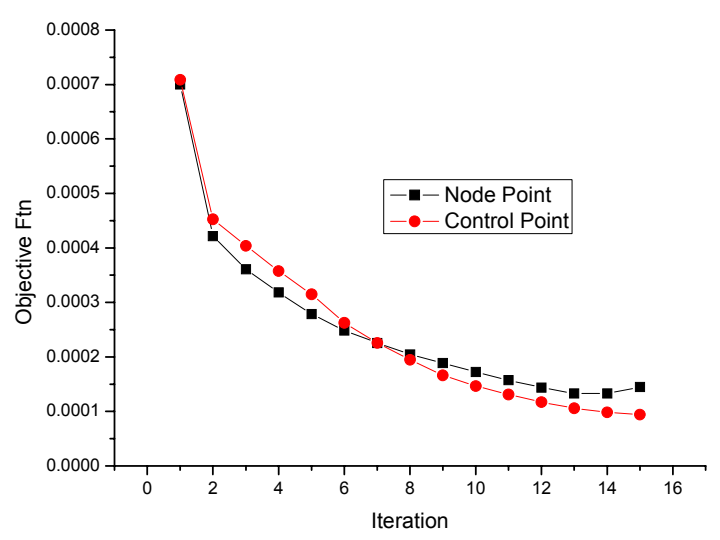

Fig. 9. Comparison of the objective function convergence

\section{Conclusion}

A new unified design environment for electric machines was developed based on CDSA and B-Spline parametrization. A commercial EM software (COMSOL) was used as a modeling tool and analysis module, and an optimization module was developed using MATLAB codes. Two modules were linked by COMSOL scripting commands to enable simplified and easy-to-use design procedure. Numerical examples show the validity of the developed design environment. Moreover, B-Spline parametrization of nodal points resulted in smoother interface boundary of the final device with better performance.

\section{Acknowledgment}

This work has been supported by KESRI (2009T100100596), which is funded by MKE (Ministry of Knowledge Economy).

\section{References}

[1] Jin-Kyu Byun, Hyun-Kyo Jung, Song-yop Hahn, Kyung Choi and Il-Han Park, "Optimal Temperature Control for Induction Heating Devices Using Physical and Geometrical Design Sensitivity," IEEE Trans. Magn., vol.34, no.5, pp.3114-3117, Sept. 1998.

[2] Dong-Hun Kim, Se-Hee Lee, Il-Han Park and JoonHo Lee, "Derivation of a General Sensitivity Formula for Shape Optimization of 2-D Magnetostatic Systems by Continuum Approach," IEEE Trans. Magn., vol.38, no.2, pp.1125-1128, Mar. 2002.

[3] COMSOL Inc. (<www.comsol.com $>$ ).

[4] Dong-Hun Kim, K. S. Ship and J. K. Sykulski, "Applying Continuum Design Sensitivity Analysis Combined With Standard EM Software to Shape Optimization in Magnetostatic Problems," IEEE Trans. Magn., vol.40, no.2, pp.1156-1159, Mar. 2004.

[5] Chang-Hyun Kim, Hyang-Beom Lee and Il-Han Park, "B-Spline Parametrization of Finite Element Models for Optimal Design of Electromagnetic Devices," IEEE Trans. Magn., vol.35, no.5, pp.3763-3765, Sept. 1999.

[6] Dong-Hun Kim, Se-Hee Lee, Il-Han Park and JoonHo Lee, "Minimization of Higher Back-EMF Harmonics in Permanent Magnet Motor Using Shape Design Sensitivity With B-Spline Parameterization," IEEE Trans. Magn., vol.39, no.3, pp.1269-1272, May 2003.

[7] DOT User Manual, Vanderplaats Research \& Development, Inc., 2001. 


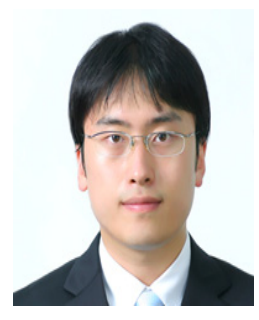

Min-Ho Kim received his B.S. degree in Electrical Engineering from Soongsil University, Seoul, Korea in 2009. He is currently pursuing his M.S. degree in the same university. His research interests include optimal design of electrical machines and numerical analysis of electromagnetic systems.

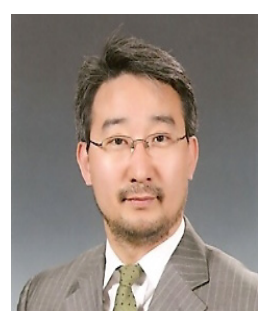

Hyang-Beom Lee received his B.S., M.S., and Ph.D. degrees in Electrical Engineering from Seoul National University, Seoul, Korea in 1989, 1991, and 1995, respectively. He is currently a Professor at the School of Electrical Engineering at Soongsil University. His research interests include nondestructive testing (NDT), heat transfer analysis of electric machines, and magnetic sensors.

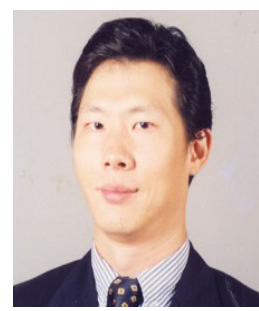

Hyeong-Seok Kim was born in Seoul, South Korea, on October 9, 1962. He received his B.S., M.S., and Ph.D. degrees from the Department of the Electrical Engineering of the Seoul National University, Seoul, South Korea, in 1985, 1987, and 1990, respectively. From 1990 to 2002, he was with the Division of Information Technology Engineering, Soonchunhyang University, Asan, South Korea. In 1997, he was a visiting professor at the Electrical Computer Science Engineering, Rensselaer Polytechnic Institute, Troy, New York USA. In 2002, he transferred to the School of Electrical and Electronics Engineering, Chung-Ang University, Seoul, South Korea as a professor. His current research interests include numerical analysis of electromagnetic fields and waves, analysis and optimal design of passive and active components for wireless communication, RFID applications, power information technology, and electromagnetic education.

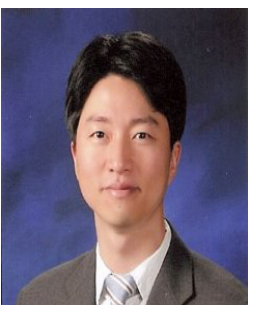

Jin-Kyu Byun received his B.S., M.S., and Ph.D. degrees in Electrical Engineering from Seoul National University, Seoul, Korea in 1995, 1997, and 2001, respectively. He worked as a Post-doc Research Associate at the Department of Electrical and Computer Engineering, University of Illinois at Urbana-Champaign from 2003 to 2005. He worked as a Senior Member of Research Staff at the Electronics and Telecommunications Research Institute (ETRI) from 2005 to 2008. He is currently an Assistant Professor at the School of Electrical Engineering at Soongsil University. His research interests include optimal design of electromagnetic systems and assessment of induced current in the human body. 